\title{
La grande séparation à résorber : L'Orient et l'Occident vus par Elisée Reclus
}

The great divide to overcome: East and West as seen by Elisée Reclus

\section{Philippe Pelletier}

\section{(2) OpenEdition}

\section{Journals}

Édition électronique

URL : http://journals.openedition.org/transtexts/185

DOI : 10.4000/transtexts. 185

ISSN : 2105-2549

Éditeur

Gregory B. Lee

\section{Édition imprimée}

Date de publication : 1 mai 2006

Pagination : 80-99

ISSN : 1771-2084

\section{Référence électronique}

Philippe Pelletier, «La grande séparation à résorber : L'Orient et l'Occident vus par Elisée Reclus »,

Transtext(e)s Transcultures 跨文本跨文化 [En ligne], 1| 2006, mis en ligne le 13 septembre 2009, consulté le 19 avril 2019. URL : http://journals.openedition.org/transtexts/185 ; DOI : 10.4000/ transtexts. 185 


\title{
La grande séparation à résorber : L'Orient et l'Occident vus par Elisée Reclus BY Philippe Pelletier
}

\begin{abstract}
Elisée Reclus (1830-1905), géographe anarchiste, a parcouru le monde entier mais ne s'est jamais rendu en Asie orientale. Grâce à d'excellentes informations récupérées grâce à son vaste et multiforme réseau de contacts, il a néanmoins beaucoup écrit sur celle-ci à la fin de sa vie. II salue l'ancienneté, sinon l'origine commune, des civilisations en Orient et en Occident, tout en déplorant leur éloignement progressif dans le temps (l'histoire) et l'espace (la séparation montagneuse et désertique du Béloutchistan jusqu'à l'Ob, en passant par les barrières de l'Hindu Kouch et des Tian Shan). Constatant leur différentiel de puissance sur la scène mondiale, qui tourne à l'avantage de l'Europe et de l'Amérique, il en recherche les causes sur le temps long et l'espace profond, en maniant ainsi une approche de géohistoire et de géopolitique avant la lettre. Sa conception géographique de l'une ou l'autre des deux aires est pionnière. Sa façon de traiter la zone-limite le distingue du futur géopoliticien Mackinder. Ses remarques sur le Japon sont à maints égards prophétiques.
\end{abstract}

\begin{abstract}
Elisée Reclus (1830-1905), an anarchist geographer, travelled round the world but never went to the Far East. Owing to excellent information collected through a wide and varied network of connections, he nevertheless wrote extensively about it towards the end of his life. He celebrated the age, if not the common origin, of Western and Eastern civilizations, while regretting their gradual separation in time (through history), and space (through the steep and desolate boundary stretching from Baluchistan to the Ob', including the barriers of Hindu Kush and of the Tian Shan). Observing that Europe and America were getting the upper hand on the world scene, he tried to determine the causes of this imbalance in history and in geography, thus implementing an early geohistorical and geopolitical method. He had a very innovative geographical conception of both regions. The way he tackled the borderline zone set him apart from the future geopolitics of Mackinder. His remarks on Japan appear in many ways prophetic.
\end{abstract}

雷克路 (Elisée Reclus) (1830-1905) 是一位无政府主义地理学家, 他的足迹几乎 遍布世界各地，却从未踏足亚洲。然而由于他庞大多重的关系网而收集的丰富 信息, 他晚年有很多关于亚洲的著作。他崇尚东西方文明的源远流长, 即使二 者不具有共同的起源, 并为它们在时间（历史）和空间（以兴都库什和天山山 
脉作为天然屏障，从卑路支到鄂毕河之间的山脉和沙漠的分隔）中的逐渐疏远 而叹惜。雷克路认识到东西方在世界势力版图上存在的力量差别, 即: 优势处 在欧洲和美洲一方，他从遥远的时间和广阔的空间领域寻找造成这种差别的 原因, 从地理历史学和地缘政治学的角度来进行研究, 突破了当时地理学科的 界限, 他对于边界地区的研究方式使之与后来的地缘政治学家麦金德（Mackinder）有 别, 而他对于日本的见解在多个方面相当有先见之明。

Elisée Reclus (1830-1905) n'est arrivé que tardivement à l'Asie orientale, si l'on peut dire. Lui qui a sillonné l'Europe, parcouru l'Amérique, du Nord comme du Sud, et visité plusieurs fois l'Afrique du Nord, ne s'y est finalement rendu qu'à l'automne de sa vie, en mars-avril 1883. Encore n'est-il, une fois dépassé Constantinople (Istanbul) et franchies les Dardanelles, guère allé plus loin que Smyrne d'après sa correspondance. ${ }^{1}$ Autrement dit, s'il a bien mis le pied en Asie, il n'a jamais visité l'Asie orientale. Voilà un premier paradoxe pour un infatigable voyageur et un esprit curieux comme lui. S'en ajoutent d'autres : l'un de ses premiers textes géographiques, sinon le premier, rédigé dans sa jeunesse lors de son premier exil (à Londres en 1852), est consacré au Japon, mais, avorté, il ne voit pas le jour ; ses écrits sur l'Asie vont se multiplier à la fin de sa vie ; et, bien que n'ayant pas visité ces espaces, il en tire des réflexions pénétrantes. $\stackrel{2}{2}$

Elisée Reclus est, comme à l'accoutumée, doté d'une vaste érudition, armé d'une méthode de travail rigoureuse, appuyé sur un vaste réseau d'informateurs, d'articles, de notes et de livres. II a puisé à de bonnes et de multiples sources concernant l'Asie orientale. Certaines sont connues, d'autres moins. Pour le Japon, il bénéficie de l'apport précieux de son collègue en géographie comme en anarchie, Léon Metchnikoff (1838-1888). Après avoir commencé des études de japonais en 1873, celui-ci a en effet vécu au Japon pendant trois ans, de 1874 à 1877. De retour en Europe, il prend, aux alentours de 1880, la suite de Gustave Lefrançais comme secrétaire d'Elisée Reclus, jusqu'à sa nomination de professeur de « Statistique et de géographie comparée » à l'Académie de Neuchâtel en 1883.

Léon Metchnikoff sera plus qu'un secrétaire pour Reclus : c'est un informateur de qualité, qui devient aussi un ami. II a notamment rédigé L'Empire japonais (Genève, 1881), « illustré de ses propres dessins originaux et bizarres, bien conçus dans le génie de la nation qu'il décrivait » comme l'écrira Elisée Reclus. ${ }^{3}$ || a puisé dans une vaste documentation, où l'on trouve les premiers grands noms de la japonologie européenne qui s'élabore à cette époque ainsi que des anciens illustres. Cet ouvrage de Metchnikoff donne de nombreux éléments

1 Elisée Reclus, Correspondance, tome II, pp. 290-304, 2 Elisée Reclus, Correspondance, tome I, p. 51. « Mon Japon, raté de plus en plus, c'était mal commencé et plus malin que je ne supposais ". Voir la note, en commentaire, des descendants Reclus compilateurs de la Correspondance: "Sans doute, un premier essai de description géographique ou politique qui, pensons-nous, ne vit jamais le jour ». 3 Elisée Reclus, « Préface » in Léon Metchnikoff, La Civilisation et les grands fleuves historiques, Paris, Hachette, 1889, pp. V-XXVIII. Dans cette très belle préface, Elisée Reclus retrace avec émotion le parcours de son ami Metchnikoff qui vient juste de décéder (pp. V-XI). II expose aussi ses conceptions de la géographie, en synergie avec le propos du livre, de façon synthétique et pugnace (pp. XII-XXVIII). Avant la préface de L'Homme et la Terre, c'est, à mon sens, l'un des textes où Reclus s'exprime le mieux à ce sujet. II est étonnant que cette préface ait été oubliée, ainsi que le livre de Metchnikoff d'ailleurs, dont le titre est certes trompeur puisque sur les onze chapitres, seul les trois derniers sont spécifiquement consacrés à la civilisation potamique. L'ensemble relève davantage d'un traité de géographie générale qui se situe pleinement dans l'optique de la géographie reclusienne. Sur Léon Metchnikoff, voir Peter Jud (1995). 
sur la géographie, la société et l'histoire du Japon. Sur les trois parties (le pays, le peuple, l'histoire), c'est la troisième, consacrée à une histoire chronologique, qui bénéficie du plus grand nombre de pages (plus de la moitié). 4

Reclus reprend les mêmes sources que Metchnikoff à propos du Japon, tout en y ajoutant d'autres. II lit les travaux des pionniers orientalistes français comme Léon de Rosny (18371914), Emile Guimet (1836-1918) ou Félix Regamey (1844-1907). II connaît les récits des différents essayistes revenus du Japon : Georges Bousquet, qui est parti enseigner le droit au Japon de 1871 à 1876 et qui en rapporte un livre très lu en France à la fin du XIXe siècle (Le Japon de nos jours et les échelles de l'Extrême-Orient, 1877) ; le voyageur allemand Rodolphe Lindau (Un voyage autour du Japon, 1864) ; le suisse Aimé Humbert (Voyage au Japon, 1866). ${ }^{5} \|$ accorde une place particulière au récit de l'écrivain britannique Isabella Lucy Bird (1831-1904), dont le titre même révèle l'originalité de cette voyageuse qui s'est rendue à cheval de Tôkyô à Hokkaidô auprès des Ainu : Unbeaten tracks in Japan (1881). Reclus cite de nombreux autres auteurs dont les noms sont familiers aux spécialistes actuels de l'Asie orientale : Alcock, Vivien de Saint-Martin, Hübner, Rein, Marton, Whitney, Müller, Von Siebold, Naumann, Oliphant, Forcade, jusqu'aux plus anciens comme le père de Charlevoix (1682-1761) ou Engelbert Kaempfer (1651-1716).

II s'appuie sur les travaux de plusieurs spécialistes en sciences naturelles qui ont exercé et mené des recherches au Japon, comme le sismologue britannique John Milne (18501913), le géologue allemand Edmund Naumann (1854-1927) (le concepteur de la fameuse Fossa magna), le géologue américain Benjamin Smith Lyman (1835-1920) ou le zoologue américain Edward Sylvester Morse (1838-1925). Plusieurs orientalistes lui transmettent des notes manuscrites : Emile Guimet, J. Serrurier, Ernest Mason Satow (1843-1929) et Basil Hall Chamberlain (1850-1935). Dans la mesure du possible, il recherche des écrits rédigés par des Japonais eux-mêmes, mais, à l'époque, les traductions sont rares.

Sur la Chine, la gamme des informations est également très large : voyageurs et explorateurs (Sven Hedin, Deniker, Escayrac de Lauture, Grenard, Forsyth, Monnier, De Rosny, Matignon, Oliphant, D'Avezac, Vigne, Bird...), ethnologues ou historiens, anciens orientalistes (Du Halde, Gaubil...) et nouveaux (Brine, Rémusat, Pelliot, Guimet, Terrien de la Couperie, Schlegel, Hervey de Saint-Denys, Ular, S. Julien, Moorcroft). II reçoit également des données directes (Richthofen, Bretschneider, Serrurier, Schiffer, Polak, Kreitner). II puise abondamment dans les livres et revues de géographie (Richthofen, Ujfalvy, Chonolky, Zakharov, Bishop).

Tout autant que par l'actualité ou la diversité des sources, le lecteur contemporain d'Elisée Reclus est frappé par leur rassemblement dans un traitement cohérent, synthétique et parfois très détaillé, vivant même alors que l'auteur ne s'est pas rendu dans les lieux. C'est particulièrement net en ce qui concerne les données plus spécifiquement géographiques où les raisonnements généraux se combinent avec des observations particulières qui, jusque là, étaient souvent éparses. Ainsi, lorsque Reclus traite de la faune et de la flore japonaises, il prend garde de replacer leur analyse dans le cadre des théories d'Alfred Wallace sur le milieu insulaire.

Bref, Reclus finit lui aussi par être fasciné par l'Asie, comme il l'est par tant d'autres choses. C'est comme si son long cheminement intellectuel et géographique l'avait finalement amené vers un débouché logique et inéluctable dans sa réflexion sur l'humanité : c'est là-bas, aussi, que la civilisation s'est développée, c'est avec elle que doit s'acheminer en conjugaison le progrès de l'humanité. $\underline{6}$

\footnotetext{
4 Léon Metchnikoff, L'Empire japonais, Genève, H. Goerg - Th. Mueller, 1881 (textes et dessins de l'auteur). 5

A signaler que ces trois ouvrages ayant été publiés par Hachette, le même éditeur que Reclus jusqu'en 1895, la recherche documentaire de celui-ci a dû en être facilitée. 6 On peut se demander si l'intérêt, ultime et non exclusif,
} 
Reclus écrit ainsi sur l'Asie orientale plusieurs textes, que l'on peut regrouper en trois séries principales : soit, chronologiquement, le volume VII de la Nouvelle géographie universelle, la terre et les hommes intitulé L'Asie orientale (1882) ; l'article inédit en français « East and West » (1894); et plusieurs passages de L'Homme et la Terre (1905) (notamment : tome I, chapitre VI, « Divisions et rythme de l'histoire »; tome III, chapitre XI, « Orient chinois »; tome V, chapitre XVII, « Les nationalités », et (livre quatrième) chapitre IV, « Russes et Asiatiques »).

Bien qu'il ne soit pas un spécialiste à proprement parler de cette partie du monde, Reclus s'impose malgré tout si l'on évoque l'Asie orientale. Les raisons d'un tel intérêt ont déjà été évoquées. ${ }^{7}$ La démarche la plus évidente consiste à tenter des rétroprojections : rapporter les descriptions de Reclus, grand géographe de la fin du XIX siècle, à ce qu'on connaît désormais des pays concernés, aussi bien à son époque qu'à la nôtre ; ou bien les comparer de façon plus intertextuelle avec celles des savants et des essayistes de son temps. Ces deux exercices cherchent à évaluer le niveau des connaissances à un moment donné pour un ou plusieurs auteurs donnés, pour tel ou tel espace. Mais ils courent le risque d'être longs, voire fastidieux, un peu vains peut-être si l'on s'en tient à l'apparence des choses qui ont tellement changé. Ils trouvent aussi rapidement des limites puisque Reclus se base sur des informations de seconde main, au minimum, et pour cause, même s'il a varié ses sources et que ses recoupements lui garantissent une grande fiabilité.

En fait, au-delà de ces deux approches, qui seront quand même esquissées à propos du Japon, il semble plus pertinent, plus ambitieux, quoique plus délicat, de cerner la logique globale, intellectuelle, scientifique mais aussi politique, au sens noble de ce terme, que Reclus mobilise pour tirer ses conclusions sur l'Asie orientale. Certes, certaines d'entre elles sont résolument datées, mais d'autres, les plus nombreuses, sont hardiment novatrices, pour ne pas dire prophétiques. Elle doivent, elles peuvent, nous aider dans notre compréhension du monde actuel, et singulièrement des rapports entre Orient et Occident, si cruciaux.

\section{Une géohistoire pertinente}

On peut légitimement considérer qu'Elisée Reclus est l'un des pionniers d'une "géographie sociale », qui porte bien ce nom $; \underline{\underline{8}}$ en cela, il se distingue de son contemporain Vidal de la Blache (1845-1918), partisan d'une " géographie humaine » (1917) qui ne signifie pas exactement la même chose. .9 On peut également dire que Reclus fait œuvre de " géopolitique ", quoiqu'il n'utilise pas ce terme qui apparaît plus tard (1916) ; en cela aussi, il se démarque de ce qui s'esquisse à l'époque chez un autre contemporain, Friedrich Ratzel (1844-1904), ancêtre

d'Elisée Reclus pour l'Asie orientale n'a pas trouvé, en partie bien sûr, un prolongement original dans le parcours d'Alexandra David-Néel (1868-1969). Alors que celle-ci commençait à s'intéresser précocement au bouddhisme et à l'Asie, à partir de 1890, elle est en effet entrée dans le cercle des Reclus et au contact d'Elisée, qui lui a préfacé son premier livre Pour la Vie (1898), un texte libertaire (réédité par les Nuits rouges en 1998), 7 Pierre Gentelle, «De la géographie physique à la géopolitique : Elisée Reclus et l'Asie orientale ", Hérodote, 22, 1981, pp. 80-93, 8 Gary S. Dunbar, "Early occurrences of the term 'social geography'", Scottish Geographical Magazine, april, vol. 93, 1977, pp. 15-20. Reproduit in Gary S. Dunbar, The History of geography, New York, Utica, 1996, p. 46-52. 9 Marie-Claire Robic, "L'invention de la 'géographie humaine' au tournant des années 1990 : les Vidaliens et l'écologie », in Paul Claval (dir.), Autour de Vidal de la Blache, la formation de l'école française de géographie, Paris, CNRS Editions, Mémoires et documents de géographie, 1993, pp. 137-147. 


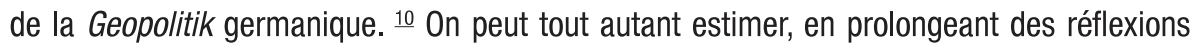
déjà engagées par quelques-uns (Gary Dunbar, Christian Vandermotten, Marie-Laurence Netter...), qu'Elisée Reclus s'affirme également comme l'un des premiers concepteurs, sinon le premier, de ce qu'on peut appeler " géohistoire », tout en soulignant que, avant tout, Reclus a réalisé une " géographie globale », pour reprendre l'expression d'Yves Lacoste. 11

Par géohistoire, on peut entendre, d'après la définition donnée par Christian Grataloup, l'étude géographique, spatialisée si l'on veut, des processus historiques, via une mobilisation des outils et des approches du géographe, pour insister sur la localisation des phénomènes socioculturels et politiques en tant que "dimension fondamentale de leur logique même ». 12 L'approche de Reclus relève totalement de cette conception. La première édition anglaise de la dernière œuvre reclusienne, L'Homme et la Terre (1905), a même pour titre Comparative Geography and History, tandis qu'en français, il avait été envisagé L'Homme à travers les contrées et les âges ou encore L'Homme, essai de géographie sociale. 13 On relèvera à nouveau la fameuse épigramme placée en tête de chaque volume de L'Homme et la Terre :

La Géographie n'est autre chose que l'Histoire dans l'Espace, de même que l'Histoire est la Géographie dans le Temps.

Reclus développe une conception originale de l'histoire : socioculturelle et géopolitique, dégagée de la chronologie événementielle. Intitulant plusieurs chapitres avec le mot histoire, il tente une progression chrono-chorologique, spatio-temporelle. C'est un exercice délicat, mais il s'avance sur un terrain en friches. II faut se souvenir qu'à la même époque les ouvrages des historiens restent en quasi totalité événementiels dans leur conception comme dans leur progression. C'est le cas, pour prendre un exemple emblématique, de l'œuvre majeure de Jules Michelet, qu'Elisée Reclus connaissait d'ailleurs personnellement, L'Histoire de la révolution française (1868).

Reclus innove, et il s'en tire à merveille car si certains passages ont naturellement vieilli ou si certaines formulations nous paraissent désuètes, son brassage des thèmes, des époques et des contrées est convaincant. Surtout, il sert son propos de fond, à savoir une étude de la civilisation humaine dans l'espace et dans le temps, avec ses diversités mais aussi ses convergences. Son souci humain et politique reste l'émancipation universelle. II cherche à comprendre ce qui a pu séparer les civilisations, ce qui les unit, ce qui peut les unir encore davantage.

L'œuvre de Reclus constitue donc une approche, avant la lettre, de temps long et d'espace profond. On peut ainsi regretter son oubli par les braudéliens, probablement imputable à la fraction des historiens marxistes. On ne fera toutefois pas la fine bouche en constatant que la relance de la géohistoire par Braudel et ses épigones a fortifié, non seulement en France mais partout dans le monde, une approche qui est en train de trouver son rythme de croisière.

La relation à l'histoire est si forte dans l'œuvre reclusienne, et si originale, qu'elle a par ailleurs perturbé les géographes vidaliens et leurs épigones qui cherchaient à ancrer leur discipline dans un paradigme étroitement spatialisé, à l'instar de la fameuse « région ». On se souvient de la fameuse phrase de Jean Brunhes (1869-1930), disciple - s'il en est - de Vidal, écrivant dans La Géographie humaine (1910) :

10 Yves Lacoste, « Géographicité et géopolitique : Elisée Reclus », Hérodote, 22, 1981, p. 14-55. Réédité dans : Paysages politiques, Le Livre de Poche, essais, 1990, pp, 191-240. 11 Lacoste, "Géographicité et géopolitique : Elisée Reclus », p. 55, 12 Christian Grataloup, "Géohistoire », in Jacques Lévy et Michel Lussault (dir.), Dictionnaire de la géographie et de l'espace des sociétés, Paris, Belin, 2003, pp. 401-402. 13 Dunbar (1978), p. 113. 
Je préfère ne pas parler ici de l'œuvre posthume de Reclus [L'Homme et la Terre] qui contient d'intéressantes vues géographiques mais qui est surtout histoire et sociologie.

Les Vidaliens ont donc décrété que tout cela n'était pas de la géographie. L'argument est assurément un prétexte mais la phrase de Brunhes est à prendre au pied de la lettre, dans toute son acception, car cela permettait de faire opportunément d'une pierre deux coups : se débarrasser de la figure populaire, mais encombrante, d'un Reclus communard, anarchiste et intellectuel libre, non universitaire, ainsi que d'une géographie reclusienne décidément trop historique, globale, trop préoccupée de questions sociales à toutes les échelles... L'échelle internationale, pour ne pas dire internationaliste ou transnationaliste, est alors probablement la plus gênante pour l'establishment à l'orée d'un XXe siècle démarrant sur fond de rivalités nationalistes, impérialistes et colonialistes, rivalités qui ne tarderont d'ailleurs pas à s'abîmer dans les guerres mondiales - la première, en 1914, n'est pas loin.

\section{Actualité de la thématique Orient-0ccident}

Les relations entre Orient et 0 ccident sont au cœur de toute réflexion sur la ou les civilisations. L'existence de l'Autre, pendant longtemps mystérieux puis de plus en plus connu, a soulevé des questions de tout ordre, philosophiques, ontologiques, existentielles, économiques, politiques, culturelles... Dans sa prétention selon les uns, son ambition selon les autres, la démarche scientifique et analytique des 0ccidentaux a régulièrement buté sur l'exemple ou le contre-exemple de l'Orient. A tel point qu'un Marx, par exemple, a dû créer une catégorie adhoc pour l'insérer dans son schéma linéaire et mécanique des stades historiques : celle $\mathrm{du}$ « mode de production asiatique ». Ce faisant, il introduit d'ailleurs l'une des rares brèches spatiales (l'Asie) dans un raisonnement qui se veut historique, économique et politique (l'Antiquité, la féodalité, la bourgeoisie...).

De fait, la continentalisation sémantique ne tardera pas à devenir un outil géographique et un projet géopolitique sous la bannière de l'européanisation ou de l'occidentalisation du monde. La difficulté analytique réside dans le fait que le binôme Orient-0ccident repose sur deux approches qui ne se recoupent qu'en partie : l'une est spatiale (un espace délimité) et l'autre notionnelle (un monde conçu). La base spatiale est supposée neutre et invariable, car vêtue du label scientifique, tandis que le notionnel partagé par l'imaginaire métagéographique de tout un chacun débouche sur des conceptualisations, des discours et des mythes. L'articulation entre les deux sert à crédibiliser des explications mais aussi à légitimer des politiques considérées comme correctes, allant de soi, logiques ou inéluctables.

Edward Said a ainsi tenté de démontrer que l'orientalisme conçu par les Occidentaux n'était, même dans son esthétisme apparemment inoffensif et son scientisme apparemment neutre, 
qu'une machine de guerre pour coloniser spirituellement et matériellement l'Orient. ${ }^{14}$ Le problème, cependant, c'est que l'Orient de Said concerne essentiellement le Proche et le Moyen-Orient, en intégrant l'Inde parfois. II en résulte un grand flou géographique et finalement conceptuel, sans parler du fait que cet Orient-là ressortit à un monde méditerranéen aux relations complexes qui le rend plus proche de l'Europe que de la Chine. En outre, un tel orientalisme dominateur n'est pas le propre des Occidentaux puisqu'il a également existé, comme viennent de l'avancer des auteurs japonais, un « orientalisme à la japonaise » qui, au cours de la première moitié du XXe siècle, légitima l'expansionnisme nippon à travers des recherches ethnographiques et géographiques. ${ }^{15}$ Pour l'historien japonais Oguma Eiji, cette théorie comporte toutefois des limites car elle n'explique pas l'émergence parallèle d'un asiatisme au Japon, qui privilégia la communauté asiatique d'intérêts face à la menace occidentale. $\frac{16}{}$

Georges Corm veut dépasser la position de Said en déconstruisant ce qu'il appelle le « mythe de la fracture Orient-Occident », non pour alimenter l'hostilité entre les deux,

mais, au contraire, pour la réduire, montrer son inanité, son rôle néfaste dans les inconscients collectifs qui s'abreuvent, en dépit de tous les progrès supposés de la laïcité, aux archétypes bibliques, qu'ils soient juifs, chrétiens ou musulmans. 17

De façon stimulante et convaincante, il montre comment l'image construite par chacune de ces civilisations, pour elle-même et vis-à-vis de l'autre, est largement fausse, car sélective, et qu'elle s'alimente symétriquement dans l'auto-mensonge réciproque. On peut rejoindre cette critique d'une «fracture imaginaire » qui allume de funestes passions, relancées par les événements du 11 septembre 2001 et l'invasion états-unienne de l'Irak, à ceci près que la partition entre Orient et 0 ccident ne passe géographiquement pas là où Corm la considère, ce qui le met d'ailleurs, et à son insu de surcroît, en contradiction avec son propos. II fait en effet commencer l'Orient à la Turquie et au Liban, alors que son raisonnement même le conduit, fort justement comme nous le verrons avec Elisée Reclus, à apparier l'Europe au monde arabo-musulman, turc et persan. Du coup, Georges Corm est poussé à ressusciter au passage un Extrême-orient comprenant la Chine et le Japon, tandis que l'Inde est évaporée. Cette question de délimitation et de définition entre Orient et Occident n'est pas anodine. Elle reste en tout cas d'une brûlante actualité car les positionnements identitaires s'accompagnent de projets politiques et économiques. La renaissance de la puissance chinoise et l'ouverture du marché chinois suscitent craintes et convoitises chez les élites occidentales, qui s'inquiètent ou se réjouissent simultanément de l'apparent marasme japonais. La constitution d'une région asiatique du type «Asean +3 » (les dix pays de l'ASEAN plus la Chine, la Corée du Sud et le Japon) qui est en branle apaise ou effraie.

\footnotetext{
14 Edward Said, L'Orientalisme : L'Orient créé par l'Occident. Paris, Le Seuil, 1980 [1978]. 15 De façon générale, I'historiographie japonaise récente s'intéresse beaucoup aux rapports entre science et colonisation japonaise. Jan Van Bremen, Shimizu Akitoshi (dir.), Anthropology and colonialism in Asia and Oceania. Londres, Curzon, 1999. Tomiyama Ichirô, "Colonialism and the sciences of the tropical zone: the academic analysis of difference in 'the Island Peoples' ", Positions, 3-2, 1995, pp. 367-391. Kevin M. Doak, "Colonialism and ethnic nationalism in the political thought of Yanaihara Tadao (1893-1961)", East Asian History, 10, 1995, pp. 79-98. 16 Oguma Eij, "Nihonjin » no kyôkai - Okinawa, Ainu, Taiwan, Chôsen, shokuminchi shihai kara fukki undô made [Les frontières des Japonais - Okinawa, Ainu, Taiwan, Corée, de la domination coloniale au mouvement pour la rétrocession], Tôkyô, Shin'yô-sha, 1998, pp. 6-7, 17 Georges Corm, Orient-Occident, la fracture imaginaire, Paris, La Découverte, nouvelle édition avec postface inédite (première édition 2002), 2005, p. 129.
} 
Chez les populations d'Asie orientale, l'Occident fascine moins qu'il y a trois décennies mais il reste attirant ou inquiétant. L'asiatisme - idéologie et/ou mouvement qui prônent le regroupement des Asiatiques - y est zébré par une histoire longue de plus d'un siècle, déjà, et très tumultueuse. Si, dès la fin du XIXe siècle, les intellectuels locaux ont pu s'entendre sur le principe de "l'Asie aux Asiatiques ", la façon de l'interpréter et la question du leadership ont divisé les élites, en particulier entre un Japon aux dents longues et une Chine forte de son aura historique. $\stackrel{18}{ }$ Ce n'est donc pas le moindre des paradoxes que de voir, à la fin du XXe siècle, dans le sillage de l'émergence des NPIA, les dirigeants de Malaisie et de Singapour en appeler au Japon pour prendre la tête d'un nouvel asiatisme, rôle que les dirigeants japonais ont décliné. Les intellectuels chinois reprennent à leur tour le chemin de l'asiatisme, tout en cherchant, chez certains, à dépasser la dichotomie Orient-0ccident. 19 Quant à la Russie, qui ne cède qu'à peine d'un pouce sur les litiges frontaliers à l'est de la Sibérie, l'ancien courant de l'eurasisme y réapparaît. 20

Les grilles d'analyse liées à ladite guerre froide ou au marxisme ayant volé en éclat, d'autres apparaissent, dominées par une logique dite culturelle à l'instar de Samuel Huntington qui interprète désormais le monde comme traversé par un "choc des civilisations". 21 On sait tous les problèmes à la fois scientifiques et politiques que soulèvent les critères civilisationnels formulés par Huntington et sa typologie, mais il faut insister sur le traitement spécifique que celui-ci réserve à l'Asie. Tandis que l'Europe occidentale et l'Amérique du Nord sont généreusement dotées des deux côtés de l'Atlantique Nord d'une même "civilisation occidentale ", l'Asie qui s'étend à l'est de l'Afghanistan se voit en effet désunie, morcelée en pas moins de cinq civilisations : « hindouiste », « bouddhiste » (qui est de surcroît représentée, sur le planisphère huntingtonien, comme étant la seule civilisation spatialement discontinue), " islamique » (Malaisie et Insulinde, au même titre que la péninsule arabique), "chinoise " (qui inclut la Corée et le Viêt-nam) et, enfin, « japonaise ». Outre l'émiettement, remarquons que le Japon est, au contraire, le seul Etat-nation à se voir doté d'une civilisation à lui tout seul, la " civilisation japonaise ", ce qui est plausible en soi (je parlerais plutôt de civilisation japonésienne), mais qui s'explique ici davantage comme une récompense pour cet allié fidèle des Etats-Unis depuis $1945 \ldots$

\section{Géohistoire et métagéographie des civilisations}

Tous ces thèmes - identités culturelles, caractères des Etats-nations, ambitions civilisatrices, guerres plus ou moins saintes - ont été abordés par Elisée Reclus. Bien sûr, il n'est pas le seul à le faire de son temps, d'où le principe de le comparer aux géographes qui lui sont contemporains dans une perspective globale.

18 Philippe Pelletier (dir.), Identités territoriales en Asie orientale. Paris, les Indes savantes, 2004. 19 Wang Hui, "Les Asiatiques réinventent l'Asie », Le Monde diplomatique, février 2005, pp. 20-21, 20 Marlène Laruelle, «Les idéologies de la 'troisième voie' dans les années 1920, le mouvement eurasiste russe ", Vingtième siècle, revue d'histoire, 70, 2001, pp. 31-46, 21 Samuel P. Huntington, "The Clash of Civilizations", Foreign Affairs, 72-3, 1993, pp. 21-49; The Clash of Civilizations and the Remaking of World Order, New York, Simon and Schuster, 1996 [trad.] Le Choc des civilisations, Paris, Odile Jacob, 1997. 
L'article East and West (1894) inédit en français, constitue une excellente synthèse de ce qu'Elisée Reclus pensait de la relation Orient-Occident. II le rédige en profitant d'une offre proposée par la revue britannique The Contemporary Review. Cette revue fondée en 1866 aborde à l'origine des thèmes religieux, politiques et littéraire, et qui traite de nos jours des sujets d'actualité. 22 Reclus y reprend des idées déjà formulées dans la Nouvelle Géographie Universelle, et qu'il exploitera à nouveau dans L'Homme et la Terre. II s'agit d'un article d'une douzaine de pages, denses, sans cartes ni illustrations mais dont on trouve celles qui lui correspondent dans le chapitre VI de L'Homme et la Terre. ${ }^{23}$ Ce texte de Reclus peut être mis en regard avec un article de même calibre et publié à la même époque par Halford John Mackinder (1861-1947) : The Geographical pivot of history (1904), qui deviendra célèbre comme référence géopolitique. $\underline{24}$

Bien que Mackinder soit le cadet de Reclus d'une trentaine d'années, les deux hommes se sont côtoyés au cours des années 1880-90 à Londres au sein de la Royal Geographical Society, aux côtés de Pierre Kropotkine (1842-1921) et d'autres.25 Mais ni l'un ni l'autre ne se font référence. Ils se rejoignent sur un point en se distinguant de Friedrich Ratzel (1844-1904), qui est, lui, quasiment de la même génération qu'Elisée Reclus. ${ }^{26}$ La Politische Geographie (1897) de Ratzel, ouvrage qui se situe également à la même époque, réfléchit en effet essentiellement sur des principes politiques (pouvoir, possession, guerre, commerce...) ou des principes spatiaux (la mer, la terre, les îles, les continents...). En revanche, les articles de Reclus et de Mackinder abordent aussi des découpages zonaux et régionaux, en tentant une spatialisation à toutes les échelles des phénomènes historiques. Mais, à part cela, leur approche, leurs analyses et leurs points de vue diffèrent radicalement.

Mackinder raisonne en termes de puissance et de pouvoir. A la conclusion de son texte (qui est la transcription d'une conférence donnée à la Royal Geographical Society le 25 janvier 1904), il déclare « avoir parlé en tant que géographe » mais il aisé de constater, en lisant l'ensemble, qu'il se pose comme un expert désireux d'orienter convenablement la politique de l'empire britannique. C'est ce que confirmera d'ailleurs sa carrière politique et diplomatique ultérieure. Cet état d'esprit apparaît bien lorsque Mackinder termine son article par une mise en garde contre la menace que représenterait « le péril jaune pour la liberté du monde » si, par malheur, « les Chinois, par exemple, organisés par les Japonais, renversaient l'empire russe et conquéraient son territoire ».

\footnotetext{
22 Elisée Reclus a écrit en tout cinq articles pour The Contemporary Review : 1. "Anarchy : by an anarchist", 45-5 (1884) ; 2. "East and West", 66-346 (1894) ; 3. "The Evolution of Cities", 67-2 (1895) ; 4. "Russia, Mongolia, and China", 67-5 (1895) ; 5. "The Progress of Mankind", 70-? (1896). D'après son livre de comptes personnel (archives de l'Institut français d'histoire sociale, Fonds Elisée Reclus, 14 AS 232, dossier IV), Reclus a reçu 342 francs de l'époque pour son article "East and West". Je remercie chaleureusement le professeur Gary Dunbar pour l'ensemble de ces informations. 23 Ainsi les cartes suivantes: "Territoires du Soleil levant au Soleil couchant", "Civilisations successives et leurs aires d'influence ", "Quelques méridiens initiaux", "Zone de dépopulation entre I'Orient et l'Occident », " Migrations océaniennes », "Routes divergentes d'Extrême-Orient », "Convergences des routes de l'Asie antérieure ", "Quelques routes de la civilisation eurasienne ", "Quelques routes de la civilisation mondiale jusqu'au voyage de Magellan-Del Cano » (avec une projection Van den Grinten)... 24 Halford John Mackinder, "The Geographical pivot of history ", The Geographical Journal, XXIII-4, 1904, pp, 421-444. Reproduit in extenso et en fac-similé dans The Geographical Journal, 170-4, décembre 2004, revue qui consacre un numéro spécial à Mackinder. Cf également : Nicolas-O, et G. Guanzini, Halford John Mackinder, géographie et politique, espace, science et géographie. Lausanne, Eratosthène-Méridien 2, 1988, qui reproduit de larges extraits de cet article pp. 38-41. A noter que Mackinder n'utilise pas le terme de géopolitique, 25 Gerry Kearns, "The political pivot of geography", The Geographical Journal, 170-4, 2004 , pp. 337-346. 26 Friedrich Ratzel, La Géographie politique, les concepts fondamentaux. Avant-propos de Michel Korinman, choix de textes et traduction par François Ewald, Paris, Fayard, 1987.
} 
Outre le fait que cette posture soit étrangère à ses convictions, Reclus raisonne au contraire en termes de civilisation. Par ce mot, il entend l'avancée d'une société. Conformément à son principe de progrès et de régrès, emprunté au philosophe napolitain Giambattista Vico (16681744), il sait aussi que les civilisations connaissent des phases de rayonnement comme de déclin et que, par conséquent, nulle civilisation n'est supérieure en soi. Bien placé aux côtés de son frère Elie Reclus (1827-1904), qui a beaucoup écrit en faveur de ce qu'on appelait alors les "peuples sauvages", Reclus se fait ainsi, systématiquement, le porte-voix des ethnies minoritaires, en les décrivant, en dénonçant leur oppression ou leur « extermination ». ${ }_{27}$ A propos du Japon, il consacre plusieurs pages aux Ainos (Ainu), très bien documentées et illustrées de dessins ou de photos. $\frac{28}{.}$

Pour Reclus, sans aucun doute, les civilisations orientales et occidentales relèvent du même monde : l'Ancien, l'Ancien Monde [ "Ancient World ", expression moins péjorative que celle d' " Old World » utilisée par Mackinder). C'est pourquoi il n'utilise pas le terme de " fracture » entre les deux, que critiquera par exemple Georges Corm, mais plutôt celui de "séparation ", de " contraste », de "distinction » ou de " divergence ». Ce choix sémantique souligne l'idée d'une origine commune de civilisation avant que "l'évolution humaine [ne] s'accomplisse différemment des deux côtés » (E\&W, p. 478).

L'Inde, notamment, est considérée comme la matrice des grandes religions, même si cellesci se sont dissociées par la suite, alors que Mackinder oppose nettement, deux par deux, puis une à une, bouddhisme, brahmanisme, mahométanisme et christianisme. Reclus se gausse de ceux qui postulent une différence de race et d'esprit entre Orientaux et Occidentaux : « Certains écrivains s'abandonnant aux fantaisies mystiques et arguant d'une prédestination supposée providentielle, ont essayé d'expliquer le contraste entre l'Ouest et l'Est par une différence de races originelle et indestructible. Les races orientales et occidentales, disentils, auraient été créées différemment, dès les commencements, l'esprit des Orientaux, nuageux et chimérique, étant porté aux perceptions tordues, aux raffinement subtils et aux ambiguïtés contradictoires, tandis que l'esprit des Occidentaux aurait été doué du génie de l'observation, d'une rectitude naturelle de pensée, de la vraie compréhension des choses. Le mythe du Serpent dans le Paradis Terrestre [the Garden], symbolisant aux yeux de ces écrivains l'influence pernicieuse de l'Orient dominerait l'histoire. Mais une conception pareille ne repose évidemment que sur le souvenir des conflits qui eurent lieu à certaines époques... $»(E \& W$, p. 483).

Bien entendu, Reclus voit bien, et il l'écrit, que la civilisation occidentale est en pleine phase d'expansion, en particulier aux dépens de la civilisation orientale. Cette dernière, toutefois, est celle qui offre le plus de résistances, contrairement à l'africaine et à l'amérindienne. L'expansion occidentale se fait sur la base d'un double processus, combiné mais contradictoire : le progrès technique, machinique et sanitaire, que Reclus soutient ; et l'impérialisme, via la guerre et les conquêtes commerciales, que Reclus critique. Mais il existe aussi à l'intérieur même des civilisations, et des sociétés, des tensions contradictoires entre les dirigeants et le peuple, l'Etat et l'association libre. La colonisation, surtout occidentale à l'époque, est le fruit de ces tensions : l'installation de populations miséreuses venues d'Europe sur de nouvelles terres à cultiver (colere en latin, qui donne colonisation) est récupérée par l'impérialisme des grandes puissances, au détriment des populations locales mais aussi des colons populaires eux-mêmes. Le colonialisme européen en Asie méridionale et orientale est toutefois différent de celui qui touche l'Afrique ou l'Amérique puisque la colonisation de peuplement reste

\footnotetext{
27 Andrée Despy-Meyer, "Elie Reclus, un ethnologue et un mythologue méconnu », Les Amis de Sainte-Foy et de sa région, 84-2, 2004, pp. 8-20. Je remercie Danièle Provain de m'avoir communiqué cette référence. 28 NGUAO, pp. 749-757; H\&T, III, pp. 91-93; H\&T, V, p. 504 et p. 527.
} 
proportionnellement faible, même dans l'Inde britannique, même dans l'Indochine française ou l'Insulinde néerlandaise.

\title{
L'identification des espaces orientaux
}

\begin{abstract}
La relativisation historique des civilisations chez Reclus trouve sa correspondance dans une relativisation géographique. Chaque ethnie puis chaque civilisation se considère naturellement, logiquement et légitimement comme étant au centre de la terre. Même l'Europe moderne et contemporaine éprouva de la difficulté à choisir son méridien de référence. Partant, chaque civilisation positionne différemment l'Est et l'Ouest. L'Asie mineure qui était l'Occident pour les Babyloniens devient ainsi l'Orient pour les Hellènes. II en découle une grande variation toponymique, question géographique par excellence, et cruciale.

Etudier les noms de lieux, de pays, constitue, rappelons-le, un domaine fondamental. Dans cette démarche, nul péché de nominalisme, doctrine qui considérait, des stoïciens à Hume en passant par Guillaume d'Occam (XIVe siècle), les espèces comme n'existant que dans les mots. Que ce soit la Moldavie ou la Transnistrie, la Palestine ou Israël, les géographes sont bien placés pour savoir ce que la toponymie recèle d'enjeux. Ainsi en va-t-il, en Asie orientale, pour la «mer du Japon » que savants et décideurs coréens aimeraient rebaptiser en «mer de l'Est ». 29 L'analyse des toponymes relève ainsi de cette métagéographie que constitue l'ensemble des structures spatiales à travers lesquelles les individus et les sociétés ordonnent leur connaissance géographique du monde. ${ }^{30} \mathrm{~A}$ cet égard, la métagéographie ne se confond pas avec la seule géographie des perceptions et des représentations puisqu'elle est aussi une façon opératoire, une géographie en acte, un instrument, un moyen qui modifie la carte même du monde.

A la rigueur, peu importent les limites et donc les dénominations pour un Reclus qui rechigne toujours à séparer les peuples. Mais il doit néanmoins les étudier pour comprendre la situation, puisque
\end{abstract}

d'un point de vue historique, il apparaît cependant utile d'essayer de déterminer approximativement la ligne normale de séparation entre les deux moitiés de l'Ancien Monde qui correspondent le mieux aux noms d'Est et Ouest. (E\&W, p. 476)

L'identification spatiale des deux civilisations orientales et occidentales ne pose toutefois pas, au préalable, de problème pour Reclus. D'un côté, il englobe l'Occident dans un vaste monde euro-méditerranéen, qui comprend le monde arabe, la péninsule arabique, le ProcheOrient, la Perse mais aussi l'ensemble de l'Afrique du Nord de l'Egypte au Maroc (ce qu'il

\footnotetext{
29 Philippe Pelletier, « Tumulte des flots entre Japon et Corée - A propos de la dénomination de la 'mer du Japon'», Annales de Géographie, 613, 2000, pp. 279-305; "The French Cartographers and the naming of the sea between Korea, Japan and Siberia during XVIlth and XVIIlth c.", The Eight International Seminar on the Naming of Seas - Special Emphasis concerning the North Pacific Ocean, Vladivostok, July 2002, pp. 16-45. 30 Martin W. Lewis, Kären E. Wigen, The Myth of continents - A critique of Metageography, University of California Press, 1997.
} 
appelle « Egypte, Cyrénaïque, Mauritanie ») (E\&W, p. 476). De l'autre côté, il englobe l'Orient, avec l'Inde, l'Indo-Chine, et l'Asie orientale qu'il définit comme étant composée de la Chine, du Japon et de la Corée.

C'est là une autre différence majeure avec Mackinder. Non content de rester assez superficiel sur l'Orient, celui-ci s'évertue à dissocier le monde mahométan du monde chrétien, donc de l'Europe stricto sensu, et à diviser ce monde mahométan en deux sous-ensembles antagonistes, l'un dominé par les Sarrazins (les Arabes), désormais assagis et contrôlables, et l'autre par les Touraniens (Turcs et turquisants), encore trop inquiétants car instables. La part belle est faite à l'Europe, chrétienne et unie, ouverte sur l'aventure atlantique et outremer : la Méditerranée n'existe pas chez Mackinder, sinon comme espace de morcellement en soi. Mackinder oppose même résolument le monde grec et le monde romain, ce que lui reprocheront d'ailleurs ses commentateurs à l'issue de la conférence (Amery, Hogarth).

Reclus a une vision novatrice en confondant l'Europe et le Proche-Moyen Orient autour de la Méditerranée. D'ailleurs, si l'on se contente d'observer l'histoire de la pensée géographique, force est de constater que, de Eratosthène (Ille siècle $B C$ ) à Martin Behaim (XVE siècle $A D$ ), en passant par Ptolémée (Ile siècle AD) ou Al-Idrisi (Xlle siècle AD) et en englobant tous les antiques géographes grecs, souvent alexandrins (comme le fameux Ptolémée lui-même), soigneusement cultivés sinon améliorés par les savants arabo-musulmans et persans jusqu'à leur retour triomphal sur la scène européenne à partir du XVe siècle, il s'agit bien d'une même conception astronomique et cosmographique, en gros. En revanche, le clivage est net une fois passé en Inde et en Chine : les latitudes et les longitudes d'Eratosthène, qui calculait la rotondité terrestre, ont peu à voir avec le quadrillage, néanmoins révolutionnaire, de son contemporain géographe chinois Pei Xiu qui postulait, sans trop s'en soucier d'ailleurs car là n'était pas sa préoccupation, une terre plate.

Le traitement géographique d'une autre partie de l'Asie par Reclus s'avère toutefois obsolète, quoique admis à son époque : celui qui concerne l'Asie du Sud-Est. L'appellation «Asie du SudEst », qui renvoie de nos jours à l'espace des dix pays de l'ASEAN, n'a d'ailleurs été adoptée qu'assez récemment, à l'issue de la seconde guerre mondiale, bien que l'anthropologue J. R. Logan l'eût proposé depuis longtemps (1847). Auparavant, d'autres appellations étaient en vigueur, recouvrant d'autres approches et d'autres espaces (Emmerson 1984, Bruneau 1986, Denécé 1999, Durand 2000). Dans l'un des volumes de la première Géographie Universelle (1837), Conrad Malte-Brun (1775-1826) distingue ainsi l'Indo-Chine (qui comprend péninsule indochinoise et péninsule malaise), un néologisme qu'il propose, et l'Océanie, autre néologisme de son cru, qui se décompose en plusieurs sous-ensembles. Parmi eux se trouve la Notasie qui désignait avant lui

les îles que l'on avait coutume d'appeler grand archipel d'Asie, archipel Indien, archipel d'Orient, et dans lequel on comprenait les îles Philippines, les Moluques, Célèbes, Bornéo, Sumâtra, ou plutôt Soumâtra, Java ; en un mot, toute la chaîne que l'on appelle les îles de la Sonde. Mais le nom de Notasie, qui signifie Asie méridionale, ne convenait point à cette division : M. Lesson, qui a visité ces archipels [en 1822-1825], et qui a reconnu que toutes les côtes des grandes îles sont peuplées de Malais, a proposé avec raison un nom plus exact, en appelant cette division Malaisie. C'est l'Océanie occidentale. 
Cette appellation d'Océanie occidentale est tombée en désuétude, de même que celle d'Australasie désignant l'espace voisin (Nouvelle-Guinée, Australie, Nouvelle-Zélande), tandis que le terme de Malaisie s'est restreint à la péninsule ou à l'Etat malais. ${ }^{31}$ Reclus adopte le point de vue de Malte-Brun pour cette partie du monde, tout en le précisant. II écrit dans la légende d'une carte que

la dénomination Australasie comprend l'Australie, la Nouvelle-Zélande, les îles Fidji, la Papouasie anglaise et les îles intermédiaires (H\&T, VI, p. 31).

0
9
$\underline{2}$

A Malaisie, il préfère le néologisme d'Insulinde (1883) :

L'ensemble des terres qui se prolonge au sud-est de l'Indo-Chine, de Sumatra à la Tasmanie, constitue, malgré sa rupture en îles distinctes, un corps terrestre analogue à l'Afrique et à l'Amérique méridionale... Que sont toutes ces terres, sinon un continent brisé, prolongeant les Indes dans l'hémisphère méridional ? C'est à bon droit que l'on a donné le nom d'Insulinde aux terres équatoriales qui continuent I'Indo-Chine au milieu de l'Océan » (NGU, VIII, 14, 4).

En 1894, il ajoute :

Nous devons réintégrer dans l'Ancien monde les îles de l'océan Indien qui font cortège aux péninsules gangétiques, et tous les groupes d'îles qui s'étirent sur l'immensité maritime vers l'est, vers l'Amérique [eastward towards America], puisque, par les migrations et les contremigrations de leurs habitants, par leurs légendes et leurs traditions, et par tout le témoignage de leur évolution historique, ces territoires océaniques forment du reste une partie du même cercle que l' "Outre-Asie » [Farther Asia] (E\&W, p. 476).

Cette conception spatiale, qui prolonge la tradition des «Indes orientales » remontant aux Grandes découvertes, ne semble plus d'actualité mais il faut noter que, de nos jours, un anthropologue célèbre comme Donald K. Emmerson propose encore une Asie du Sud-Est comprenant Ceylan, Andaman, Nicobar, Assam, Yunnan, Hainan mais excluant les Célèbes ou les Philippines qu'il fait appartenir à la « zone Pacifique ».32

Le second extrait permet de montrer la logique de Reclus : il y a insertion des espaces et des peuples dans la même aire de civilisation par le jeu des dynamiques migratoires et des histoires communes. C'est le mouvement, l'échange, qui crée l'identité, et non le seul lieu de naissance. De fait, Reclus trace deux grands mouvements historiques de civilisation, l'un vers l'est, les mers de Chine et le grand Océan (Pacifique), l'autre vers l'ouest, vers la Méditerranée et l'autre océan, l'Atlantique. C'est à la civilisation qui parviendra à franchir ces océans, et donc à gagner l'Amérique, que reviendra un avantage décisif : sur ce point, Reclus et Mackinder sont évidemment d'accord.

31 Yves Lacoste, "Editorial : Australasie », Hérodote, 52, 1989, pp. 3-14. 32 Donald K. Emmerson, "'Southeast

Asia' : What's in a Name?", Journal of South Asian Studies, XV, 1, 1984, pp. 1-21. 


\section{A la recherche de la «zone médiane» (Reclus) ou du «pivot» (Mackinder)}

Reclus cherche donc l'espace de séparation entre Orient et Occident puisque celui-ci nous permettra de comprendre, au moins en partie, l'évolution respective de chaque civilisation orientale et occidentale. Reclus et Mackinder semblent se rejoindre sur ce point puisqu'il vont traiter tous les deux de l'Asie centrale. Mais leurs approches s'opposent et, dans le détail, l'espace considéré n'est en réalité pas le même.

Mackinder expose sa théorie du " pivot », qui deviendra le fameux « Heartland », cœur du Monde que les puissances doivent contrôler pour assurer leur domination. II situe celui-ci à la hauteur des steppes d'Asie centrale, mais plutôt en bordure de la zone méridionale de la Sibérie, où l'influence russe est déjà acquise. Pour lui, cette zone de steppe est propice aux nomades, fatalement esclavagistes et conquérants, comme les Huns ou les Mongols, de toute façon menaçants sinon barbares. C'est la réalité climatique de cette steppe qui conditionne une telle évolution. Elle s'oppose à l'ancestrale forêt de la Russie et de l'Europe occidentale, défrichée depuis et transformée en plaines agricoles de sédentaires.

En revanche, Reclus définit la «ligne de séparation " entre Orient et Occident comme étant une "zone médiane » essentiellement caractérisée par le «nœud central », le "Toit du monde " (E\&W, p. 328), que sont les chaînes de montagnes de l'Hindu Kuch, du Karakorum, de l'Himalaya et du plateau tibétain. Ce sont les difficultés de passage (cols élevés et peu praticables, gorges, déserts...) et d'échange qui sont d'abord responsables de la non-communication entre le monde sinisé ancien et le monde gréco-romain, malgré les tentatives par le sud (Alexandre), voire en évitant les obstacles du désert bélouchistanais et des montagnes afghanes par l'emprunt de la route maritime.

De surcroît, les grands fleuves ont favorisé l'orientation vers l'est en Asie (Gange en Inde, Yangzi et Huang-He en Chine), en direction du grand océan, tel le "diaphragme de l'Asie " (E\&W, p. 331). Le monde insulindien relève d'abord de I'Inde selon Reclus. Le monde polynésien part de cette Insulinde malaise, comme il l'explique dans un long passage original où il révèle sa nouvelle passion pour l'Océanie, une Océanie, répétons-le, rattachée à l'Asie (E\&W, p. 330-482). Par contre, en Occident, certains fleuves et surtout la mer Méditerranée ont favorisé un tropisme latitudinal, de plus en plus dirigé vers l'ouest.

II semblerait donc que Reclus ait abandonné, au moins dans cet article de 1894 et dans le chapitre concerné de L'Homme et la Terre, la théorie de la dessiccation de l'Asie centrale, telle qu'il l'avait exposée dans le volume Asie orientale de la NGU (1882). Selon cette théorie, en partie validée par les découvertes scientifiques récentes, un desséchement du milieu aurait provoqué des migrations précoces de population et le dépeuplement de cette zone. Mais Reclus n'en parle plus. 
S'agissant du rapport des sociétés à l'environnement sur les espaces terrestres, on observe donc une tendance à l'influence climatique chez Mackinder, dans un mode quasi déterministe que l'on retrouvera d'ailleurs souvent chez les géographes anglo-saxons (Ellen Churchill Semple, Ellsworth Huntington...), tandis que le tropisme tellurique, fort chez Reclus, échappe au piège déterministe : en effet, si l'être humain peut difficilement modifier le climat, constat sur lequel s'appuie le déterminisme géographique pour postuler des causalités inamovibles sur la socioculture, il peut surmonter les obstacles imposés par le relief grâce à des routes, des tunnels, des paravalanches et divers équipements. L'être humain peut s'affranchir de cette difficulté physique.

En outre, Reclus relativise simultanément le rôle du relief avec celui des bassins fluviaux, qui lui est lié. Certes, il souligne régulièrement que les anciennes civilisations sont nées au bord des grands fleuves (Mésopotamie, Egypte du Nil, Chine du fleuve Jaune, Inde de I'Indus et du Gange). Mais il ne considère pas que leur débouché maritime soit strictement déterminant puisqu'il faut le replacer dans un contexte plus global, " aussi bien sous le rapport de l'histoire que de son orientation géographique [générale] » (E\&W, p. 478). Dans cette perspective dynamique, il souligne le contraste des occupations démographiques, bien représenté sur une carte des densités où la "zone médiane » très peu peuplée sépare Occident et Orient beaucoup plus peuplés.

De fait, là où Mackinder craint l'extension des réseaux ferroviaires à travers les steppes ou les plaines sibériennes, Reclus se réjouit de tout aménagement qui permet une circulation entre les hommes, libre et meilleure. II s'agit, selon lui, d'un gage de fraternité des peuples, un principe que l'on retrouve dans toute son œuvre. Même si on peut lui contester un certain optimisme sociologique et philosophique sur ce point, du moins faut-il reconnaître que la libre circulation constitue une condition sine qua non du progrès social : c'est d'ailleurs comme cela que Reclus l'envisage. D'où sa conclusion

\footnotetext{
Il a été dit une fois - quoique dans un sens purement dynastique, que l'histoire n'a pas encore ratifié - que « les Pyrénées n'existent plus »! || reste à la civilisation occidentale de dire, véritablement, d'un point de vue humain et non dynastique : "Nous en avons fini avec l'Himalaya» (E\&W, p. 487).
}

Ce que veut enfin savoir Reclus, c'est pour quelles raisons - d'abord intrinsèques, venant de l'intérieur, du lieu lui-même - la civilisation orientale a décliné au profit de la civilisation occidentale. Fidèle à sa dialectique de milieu-espace et de milieu-temps qu'il exposera avec brio dans la préface de L'Homme et la Terre, il analyse diachroniquement et synchroniquement les espaces concernés.

Selon lui, les causes principales du retard pris par l'Orient sur l'Occident, manifesté par la puissance technologique, économique et politique de celui-ci, sont au nombre de trois : l'immensité de son débouché océanique vers l'est, qui amène les sociétés orientales à tourner en rond ou à se perdre, tandis que l'Occident passe l'Atlantique (E\&W, p. 483-484) ; le caractère centrifuges des axes développement, en particulier le long des fleuves qui ne se rejoignent pas, d'où l'absence d'une unité politique précoce, forte et stable, malgré quelques épisodes, d'où l'émiettement des bassins de civilisations (Indus, Gange, Fleuve jaune...) ; l'absence d'un « centre géographique commun », notion qui se distingue en réalité 
du pivot selon Mackinder, absence renforcée par l'existence, en plusieurs points, de barrières de séparation, notamment là où devrait être le centre (du Bélouchistan au Tibet). Au contraire, à l'Ouest, les veines de civilisation

au lieu de diverger dans un angle obtus, tendent l'une vers l'autre, toutes convergeant uniformément vers le bassin de la Méditerranée hellénique (E\&W, p. 485).

Même si Reclus constate le déplacement vers l'Europe occidentale et septentrionale du " centre de gravité » de la civilisation européenne, le cadre général reste tracé ( $E \& W$, p. 486). "Le domaine sans cesse croissant de l'ascendance européenne finit par embrasser le monde entier ", jusqu'au... Japon, situé de l'autre côté. Japon : " La civilisation n'aura point à déplorer l'asservissement honteux de quarante millions d'hommes » (NGU, VII, p. 772).

Le Japon confirme ce que Reclus pense de la marche du Monde. Analyser l'évolution de ce pays à partir de Meiji en termes d'imitation servile, pure et simple de l'Occident est, selon Reclus, une stupidité, un orgueil narcissique mal placé chez les Occidentaux ainsi qu'une erreur empêchant d'évaluer correctement les phénomènes. Au-delà des descriptions si fouillées et si vivantes, incroyables pour quelqu'un qui n'a même pas mis les pieds dans le pays, fondées sur une série impressionnante d'informations, s'il y a un bilan à retirer de l'analyse reclusienne sur le Japon, c'est celui-ci.

L'introduction du chapitre sur le Japon dans la Nouvelle géographie universelle donne ainsi le ton :

[c'est] un des pays les plus curieux de la Terre par sa nature, ses habitants, son histoire, et surtout par les événements qui s'y accomplissent. De toutes les nations vivant en dehors de l'Europe, du Nouveau Monde et de l'Australie, les Japonais sont les seuls qui aient accueilli de plein gré la civilisation de l'Occident, et qui cherchent à s'en appliquer toutes les conquêtes matérielles et morales. Ils n'ont pas eu, comme tant d'autres peuples, le malheur de perdre leur indépendance, et la force ne leur a pas imposé les mœurs d'une nation victorieuse ; l'ascendant d'une religion étrangère ne les a pas non plus groupés comme un troupeau sous les lois de leurs convertisseurs. Libres politiquement et religieusement, c'est en qualité de disciples volontaires, et non de sujets, qu'ils entrent dans le monde européen pour lui emprunter ses idées et ses mœurs» (NGU, VII, p. 686).

Quoiqu'encore entaché d'un européocentrisme presque naif, ce propos dégage l'essentiel : l'indépendance, la liberté politique et religieuse sont les gages du progrès, au Japon comme ailleurs.

Tandis que l'essayiste Georges Bousquet pronostique la superficialité du changement et redoute un réveil de la barbarie japonaise sur fond de péril jaune, Reclus, comme le constate Gérard Siary,

ne dissimule pas son admiration pour un peuple qui ne se laissera pas conquérir sans lutte (...) et rétorque qu'il est impossible qu'une nation revienne sur les progrès accomplis quand ceux-ci s'appuient sur un développement scientifique réel.

Et Siary de conclure : 
Dans l'ensemble, pour tenu qu'il soit de faire la part entre des témoignages parfois contradictoires, le géographe Reclus exploite l'ethnographie au profit des Japonais. C'est l'anti-Bousquet français. $\frac{33}{}$

A l'instar de la quasi totalité des observateurs européens des siècles précédents, de François Xavier (1506-1552) à Von Siebold (1796-1866), en passant par Kaempfer (1651-1716), Thunberg (1743-1828) et tant d'autres, Elisée Reclus loue les vertus psychologiques et morales des Japonais, leur conscience intérieure d'où n'est jamais absent le regard de l'autre :

Les Japonais sont retenus dans la voie des études et du développement qui en est la conséquence par une de leurs fortes qualités nationales, le respect de l'honneur. II se sentent engagés, et cela suffit : ils fourniront les preuves de civilisation qu'on leur demande » (NGU, VII, p. 771).

Nous verrons que ce constat aux apparences de philosophie idéaliste est corroboré par une analyse plus matérialiste du milieu géographique japonais.

L'un des aspects les plus remarquables de cette analyse, c'est que Reclus la développe précocement: en 1882, date de publication du volume VII de la Nouvelle géographie universelle, c'est-à-dire moins d'une quinzaine d'années après la Restauration Meiji (1868), donc avant même que n'aboutissent toutes les implications de celle-ci. Bien que Léon Metchnikoff n'aille pas aussi loin que Reclus, ses observations de première main ont de toute évidence été utiles pour tirer des conclusions aussi rapides que précises. On peut en déceler les prémisses dans L'Empire japonais (1881), lorsque Metchnikoff rappelle que

avant son contact avec la civilisation occidentale, le Japon possédait déjà des antécédents de longs siècles de culture qui, bien que puisée à une source étrangère (chinoise) à son origine, ne tarda pas à s'acclimater dans ce pays si richement doué par la nature, y poussa profondément ses racines et y fut marquée d'un cachet indigène d'intelligente impressionnabilité et d'aimable vivacité qui font défaut même aux nations les plus avancées du continent asiatique. 34

Voilà qui confirme Reclus dans son idée que la civilisation occidentale n'est pas supérieure par essence, même si elle a le vent en poupe.Dans l'ouvrage qui viendra une vingtaine d'années plus tard, L'Homme et la Terre, Reclus confirme son point de vue. Le changement opère au Japon, écrit-il,

de manière plus simple, plus noble et plus dramatique [qu'en Chine] : les résultats politiques et sociaux en furent peut-être, pendant le dix-neuvième siècle, la plus grande merveille de l'histoire, car il ne s'agit de rien moins que de l'arrachement d'une nation au cycle fermé de la civilisation orientale et de son entrée presque soudaine dans le monde européanisé. Evidemment pareille transformation ne peut s'expliquer que par une pression intérieure d'une puissance extraordinaire $»(H \& T, V, p .171)$.

Les changements des Japonais ne sont pas non plus superficiels, ils ne sauraient être

33 Gérard Siary, "Le discours ethnographique sur le Japon en France dans la seconde moitié du XIX siècle et au début du XXe siècle ", L'Ethnographe, LXXXVI-2, 1990, pp. 69-83, 34 Metchnikoff, L'Empire japonais, p. 11. 
"attribués à un accès collectif de vanité, à une fièvre épidémique de la mode ", ils sont «intimes» (H\&T, V, p. 527). Reclus en donne un exemple emblématique : la coutume du hara-kiri (transcription abusive du terme correct de seppuku, également donné par Reclus), qui l'a tant frappé dans la Nouvelle géographie universelle en ce qu'elle « témoigne de la force de volonté qu'ils savent mettre à la revendication de leur dignité personnelle » (NGU, VII, p. 772), est abandonnée ; les nobles japonais, dont c'était « le farouche orgueil », ont même eu « le bon esprit de ne pas [la] remplacer par le duel à la française » (H\&T, V, p. 528). Reclus modifie d'autant moins son opinion sur le Japon que les événements lui donnent largement raison :

Ce qui empêche de douter que les transformations politiques et sociales du Japon sont bien réellement des changements définitifs, ne permettant plus de retour en arrière, c'est qu'elles ont passé, pour ainsi dire, par l'épreuve du feu (H\&T, V, p. 328).

Reclus fait ici allusion au mouvement d'opposition, plus ou moins réactionnaire, d'une partie des pionniers de Meiji qui, déçus, s'étaient retournés, en vain, contre le régime (notamment la guerre civile dite de Satsuma en 1877, avec Saigô Takamori).

Elisée Reclus est donc conduit à analyser le Japon en privilégiant la dynamique endogène du pays, tout en la gardant dans le contexte régional et mondial, contrairement aux orientalistes, aux tenants de l'exotisme et à certains Japonais eux-mêmes, les "nipponistes", qui s'évertuent à considérer une essence voire une pureté japonaises, où sont minimisés tous les contacts avec le continent et l'extérieur. Cette approche reclusienne recoupe son analyse du milieu physique japonais dont il loue la richesse et la variété : «L'abondance des pluies, la modération relative des hivers et la chaleur humide des étés donnent à la flore du Japon une richesse et une vigueur extraordinaire » (NGU, VII, p. 739), à l'instar de ces "splendides cryptomérias, la gloire du pays » (NGU,VII, p. 742). Elisée Reclus est beaucoup plus fasciné par la beauté des volcans que par la dangerosité de leurs éruptions. II n'évoque que rapidement les séismes ou les typhons, et (problème d'information ?) ne dit mot des tsunamis.

L'adaptation au milieu, le caractère minutieux de l'agriculture (des "excellents jardiniers ") et de l'artisanat japonais sont des gages pour un développement industriel. Même les matières premières existent, avec l'eau, le bois, certains minerais comme les "gisements considérables de fer, d'or (...), l'extrême richesse en couches de houille » (NGU, VII, p. 828), ces richesses que négligeront les geignards du " pauvre Japon hostile et dépourvu de tout » escortant les thuriféraires du "miracle japonais » au cours des Trente Glorieuses. Reclus n'oublie pas de les placer dans le contexte évolutif des économies : "L'industrie minière du Japon est relativement moins importante qu'elle ne l'était autrefois » (NGU, VII, p. 828), c'est-à-dire par rapport à l'époque où le Japon, l'eldorado du Cipango, exportait des métaux précieux (XVle, $X V \|^{e}$ et $X V I I I^{e}$ siècles). Certes, ces matières premières ne suffiront plus au zénith de la Haute Croissance et de la pétrochimie, mais elles auront servi au décollage proto-industriel et manufacturier du pays.

Cette analyse géographique de Reclus se place en droite ligne de ses prédécesseurs européens qui louent la munificence et la beauté du milieu japonais. Ainsi le portugais Jorge Alvares salue la profusion des arbres, des fruits et des cultures (1546). Son compatriote João Rodrigues signale l'abondance des montagnes rudes mais aussi la belle culture des plaines et 
la salubrité du climat (1620). L'Anglais Arthur Hatch, qui remarque l'excès des aléas naturels, vante l'étendue du pays, l'aménagement de son territoire et le caractère tempéré de son climat (1620). Plus tard, le visiteur bavarois Engelbert Kaempfer affirme :

Le Japon peut vivre sans aucun contact avec l'étranger parce que c'est un pays très favorisé. (...) Son climat est très favorable : il rend les femmes fécondes, les terres fertiles, les maladies rares (1727).

Quant au visiteur suédois Carl-Peter Thunberg, il décrit le Japon comme un "pays d'abondance » :

Malgré sa prodigieuse population, ce royaume jouit de la plus grande abondance ; la disette s'y fait rarement sentir. (...) II n'y a peut-être pas de pays au monde plus abondant en comestibles que les îles du Japon : le sol même et les mers voisines en procurent de toutes les espèces, que l'art sait encore multiplier » (1780).

Bref, au cours de l'époque moderne, les observateurs européens sont d'accord pour souligner les bienfaits du milieu naturel japonais. Les Japonais, dont ils s'inspirent, ont le même point de vue. Ils le perdront cependant au cours du XXe siècle, entraînant avec eux les observateurs étrangers. C'est notamment le cas après la défaite de 1945, quand il fallut expliquer et légitimer idéologiquement l'idée d'un prétendu « miracle japonais » pour mieux cacher ses vraies recettes (réintégration de la technobureaucratie militaro-fasciste, recyclage d'un capitalisme d'Etat, soutien anticommuniste des Etats-Unis, répression ouvrière....). $\frac{35}{3}$ Outre le caractère parfois hétéroclite de ses descriptions dans la Nouvelle géographie universelle, au demeurant conforme au processus narratif de l'époque, l'une des lacunes de Reclus réside, paradoxalement vu ses idées, mais probablement faute d'informations, dans le manque de description des conditions laborieuses du peuple japonais en voie de prolétarisation.

Mais Reclus sait se montrer visionnaire en s'inquiétant d'une possible montée en puissance du militarisme japonais. Déjà, dans la Nouvelle géographie universelle, il termine son propos en évoquant les capacités militaires déjà suffisantes du Japon pour

braver la Chine, la Corée, et résister même à la Russie ; mais il est à craindre aussi que le Japon, fier de son armée solide, ne se laisse entraîner à une politique d'agression sur ses voisins moins faibles » (NGU, VII, p. 855).

Alors que des historiens américains contemporains ergotent pour savoir si le militarisme japonais de la première moitié du XXe siècle était déjà programmé dans le système meijien, une telle prévision, faite en 1882 répétons-le, laisse songeur... Soulignons aussi qu'il ne s'agit pas chez Reclus d'une évocation du " péril jaune " anti-occidental, dont s'empareront les essayistes européens dès le début du XX siècle à la suite de Guillaume II, et qu'agite déjà un Georges Bousquet en 1877, mais bien de l'extension du Japon aux dépens de ses voisins, les peuples asiatiques. 
Là encore, les événements vont donner raison à Reclus. Constatant un "très grand ascendant d'assimilation » des peuples voisins par les Japonais, il déplore aussi qu' « il est malheureusement facile (...) de changer des laboureurs en militaires. (...) C'est là un danger imminent en cas de nouveaux conflits » (H\&T, V, p. 525). Il craint « là-bas, du côté de l'Extrême-Orient, la guerre sourde ou déchaînée entre Japonais et Russes ", au détriment des Chinois, même s'il estime que l'emporteront « les progrès étonnants qui s'accomplissent en ces régions du monde dans le sens de la répartition de la culture et de l'idéal humain » (H\&T, VI, p. 519). II ne faudrait pas que, de nos jours, les Japonais, attirés par un nouveau nationalisme, ainsi que d'autres peuples oublient cette aspiration. 